Revista Arbitrada Interdisciplinaria KOINONIA

Año V. Vol V. N¹0. Julio - Diciembre 2020

Hecho el depósito de Ley: FA2016000010 ISSN: $2542-3088$

FUNDACIÓN KOINONIA (F.K). Santa Ana de Coro. Venezuela

Cristian Homar Blacio-Aguilar; Cecilia Ivonne Narváez-Zurita; Juan Carlos Erazo-Álvarez

http://dx.doi.org/10.35381/r.k.v5i10.687

\title{
Normas internacionales de información financiera y reconocimiento contable de jugadores de fútbol en clubes deportivos
}

\section{International financial reporting standards and accounting recognition of soccer players in sport clubs}

\author{
Cristian Homar Blacio-Aguilar \\ cristian.blacio@psg.ucacue.edu.ec \\ Universidad Católica de Cuenca, Cuenca \\ Ecuador \\ https://orcid.org/0000-0003-2832-1618 \\ Cecilia Ivonne Narváez-Zurita \\ inarvaez@ucacue.edu.ec \\ Universidad Católica de Cuenca, Cuenca \\ Ecuador \\ https://orcid.org/0000-0002-7437-9880 \\ Juan Carlos Erazo-Álvarez \\ jcerazo@ucacue.edu.ec \\ Universidad Católica de Cuenca, Cuenca \\ Ecuador \\ https://orcid.org/0000-0001-6480-2270
}

Recibido: 07 de marzo de 2020

Revisado: 20 de marzo de 2020

Aprobado: 30 de abril de 2020

Publicado: 19 de mayo de 2020

\section{RESUMEN}

La presente investigación tiene por objetivo diseñar una guía de procedimientos contables para el reconocimiento y medición de jugadores de fútbol en clubes deportivos del Ecuador. Para lograrlo, se ha empleado un enfoque cualitativo de indagación, además, una entrevista al Club Deportivo Cuenca y una ficha de revisión documental que han aportado significativamente a este trabajo y su propuesta. Los resultados de la investigación indican que los clubes no reflejan sus activos intangibles en sus estados financieros. En base a metodologías internacionales y lineamientos de la NIC 38 se ha establecido una guía para el tratamiento contable de jugadores de fútbol en sus etapas de reconocimiento y medición. El primer paso sería la revalorización de su plantilla actual, y seguir todos los procesos establecidos en este trabajo para lograr la transparencia, razonabilidad y comparabilidad que son características esenciales en la contabilidad. 
Cristian Homar Blacio-Aguilar; Cecilia Ivonne Narváez-Zurita; Juan Carlos Erazo-Álvarez

Descriptores: Contabilidad de costes; estado financiero; financiación; derecho de los contratos; competencia deportiva. (Palabras tomadas del Tesauro UNESCO).

\begin{abstract}
This research aims to design a guide of accounting procedures for the soccer players' recognition and measurement in sport clubs in Ecuador. To achieve this, a qualitative approach of inquiry has been used; as well as an interview with the Cuenca sport Club and a document review form that have contributed significantly to this work and its proposal. The results of the investigation indicate that the clubs do not reflect their intangible assets in their financial statements. Based on international methodologies and guidelines of IFRS 38, a guide has been established for the accounting treatment of soccer players in their recognition and measurement stages. The first step would be the revaluation of their current workforce followed by all the processes established in this work to achieve the transparency, reasonableness and comparability which are essential characteristics in accounting.
\end{abstract}

Descriptors: Cost accounting; financial statements; financing; contract law; sports competitions. (Words taken from the UNESCO Thesaurus).

\title{
INTRODUCCIÓN
}

La normativa contable internacional establece lineamientos para el reconocimiento y manejo de los diferentes elementos que componen los estados financieros de una empresa, mostrando la razonabilidad y realidad de las cuentas. Los activos intangibles, son elementos de patrimonio, tal como indica su nombre, se caracterizan por su naturaleza intangible, y la susceptibilidad de ser medidos económicamente.

Los activos intangibles, pueden ser generados por la empresa o resultados de una transacción onerosa, es por ello, que en la primera parte del presente trabajo se describirán las Normas Internacionales de Información Financiera (NIIF), sus antecedentes y adopción en Ecuador, posteriormente, se realizará un análisis para determinar el tratamiento contable y financiero más adecuado al capital humano de las instituciones deportivas tanto de los activos intangibles generados internamente, como de los adquiridos.

Al respecto, se evidencia diferentes terminologías en la contabilidad deportiva como por ejemplo en el caso de España, los derechos de traspaso, que se utiliza para referirse a los derechos de adquisición de los jugadores, que incluye derechos de uso y explotación deportiva de los jugadores profesionales que forman parte de la 
Cristian Homar Blacio-Aguilar; Cecilia Ivonne Narváez-Zurita; Juan Carlos Erazo-Álvarez plantilla que compite en campeonatos o ligas locales e internacionales. En países de todo el mundo se presenta el problema con el reconocimiento contable de estos activos, por cuanto existen diferentes niveles en la vida deportiva de un jugador, donde muchos de estos no llegan a formar parte de la plantilla principal de un equipo, por consiguiente, no se convierten en un activo intangible. Por otro lado, la valoración incluye muchas variables que aumentan o disminuyen el valor de un jugador al momento de un traspaso, por lo que es importante identificar y utilizar correctamente las mismas en las diferentes metodologías propuestas a nivel internacional que pueden ser utilizadas.

En el presente trabajo se analiza tanto el reconocimiento contable de los jugadores y la valoración del activo intangible, con el objeto de diseñar una guía de procedimientos contables para el reconocimiento de jugadores de futbol en clubes deportivos del Ecuador, como herramienta para el mejoramiento de la calidad de la información contable. En base a lo mencionado en los párrafos que anteceden, surge una interrogante: ¿Cuál es la metodología más adecuada para reconocer y medir correctamente a los jugadores de fútbol dentro de la información financiera de una entidad deportiva?

Existen diferentes métodos de valoración a nivel mundial que se basan en variables que se ajustan a las necesidades de cada club deportivo y a la información de la cual disponen, no obstante, es importante aplicar una metodología basada en Normas Internacionales de Información Financiera para que dichos resultados puedan ser comparables y aceptados a nivel no solo local sino internacional.

\section{Referencial teórico}

\section{Normas internacionales de información financiera y reconocimiento contable de los jugadores de fútbol en los clubes deportivos}

Las NIIF son un conjunto de normas emitidas desde el año 2000 por la Junta de Normas Internacionales de Contabilidad (IASB por sus siglas en inglés), la cual está conformada por miembros de organismos regulatorios organizaciones internacionales, corporaciones globales y de la profesión contable, quienes desarrollan y aprueban las NIIF (Ugalde, 2014). Esta normativa busca mejorar la 


\section{Revista Arbitrada Interdisciplinaria KOINONIA \\ Año V. Vol V. N${ }^{\circ} 10$. Julio - Diciembre 2020 \\ Hecho el depósito de Ley: FA2016000010 ISSN: 2542-3088 \\ FUNDACIÓN KOINONIA (F.K). Santa Ana de Coro. Venezuela.}

Cristian Homar Blacio-Aguilar; Cecilia Ivonne Narváez-Zurita; Juan Carlos Erazo-Álvarez transparecia, rendicion de cuentas, la eficiencia económica de las empresas de todo el mundo, fomentando confianza, crecimiento y estabilidad financiera a largo plazo en la economía a nivel mundial (Fundación de Estándares Internacionales de Reportes Financieros IFRS ${ }^{1}$, 2018).

De acuerdo a (Mesén, 2007) algunas de las razones más importantes para la aplicación de las NIIF a nivel internacional son: fomentar la transparencia de los estados financieros de las empresas; el proceso de globalización e integración económica; establecer un lenguaje común de lectura contable para reducir la diferencia en los principios de contabilidad a nivel mundial, entre otros. En este sentido, las diferencias que existen en las definiciones de los elementos de la información financiera de las empresas, dependen mucho de las circunstancias sociales, económicas, legales, e incluso por las necesidades que tiene cada país.

Es por ello que se pueden generar distintos criterios para reconocer y medir estos elementos y que empresas con actividades iguales que se encuentren en diferentes jurisdicciones no puedan comparar su información (Marcotrigiano, 2011). La adopción de las NIIF en Ecuador nace a raíz de que las Normas Ecuatorianas de Contabilidad (NEC), no se actualizaban a la misma velocidad de las normativas a nivel internacional, generando un problema al momento de comparar la información contable y financiera de las empresas ecuatorianas con las del resto del mundo (Pérez, Fernández, Méndez, Méndez \& Alvarado, 2015). De acuerdo a la (Superintendencia de Compañias del Ecuador, 2011), las NIIF completas son aplicadas por empresas cotizantes de 123 países en todo el mundo, por ello, se hace necesaria la adopción de esta normativa en el Ecuador (Quizhpi, Narváez \& Erazo, 2019).

Al mismo tiempo, los autores (González, Cuenca, Higuerey \& Villanueva, 2017) mencionan que Ecuador al ser un país potencialmente atractivo para inversores, necesita que sus empresas cumplan con exigencias empresariales a nivel mundial. De acuerdo a esto, la (Superintendencia de Compañias, Valores y Seguros, 2008) mediante resolución $N^{\circ}$ 08.G.DSC.010 estableció un cronograma para la implementación de las NIIF desde el año 2010 al año 2012. Con la finalidad de

\footnotetext{
${ }^{1}$ IFRS: siglas en inglés para Estándares Internacionales de Reportes Financieros.
} 
Cristian Homar Blacio-Aguilar; Cecilia Ivonne Narváez-Zurita; Juan Carlos Erazo-Álvarez mejorar y transparentar la información financiera a los diferentes usuarios, se agregaron más estados financieros a los que normalmente se presentaban; las NIIF exigen un juego completo, dentro de los cuales se encuentran: balance general, estado de pérdidas y ganancias, estado de flujo de efectivo, estado de cambios en el patrimonio y notas explicativas a los estados financieros (Espinoza, Narváez, Erazo \& Machuca, 2019).

\section{Norma Internacional de Contabilidad (NIC) 38 y el tratamiento contable de los activos intangibles}

De conformidad con la norma de contabilidad 38, se define a un activo intangible como un elemento identificable, con naturaleza no monetaria y sin apariencia física, utilizado para la producción de productos o servicios, arriendo o para propósitos administrativos. De acuerdo a la (Fundación IFRS, 2017), el alcance de esta norma incluye:

- Marcas.

- Cabeceras de medios de comunicación y sellos o denominaciones editoriales.

- Programas y aplicaciones informáticas.

- Concesiones o franquicias.

- Derechos de propiedad intelectual, patentes o derechos de explotación.

- Recetas o fórmulas, modelos, diseños y prototipos.

Al respecto (Barrios, Correa, Acosta \& González, 2003), señalan que existen dos tipos de intangibles; en primer lugar están los activos intangibles adquiridos, que son aquellos que resultan de transacciones onerosas con terceros o por combinación de empresas, por otro lado, se encuentran los activos intangibles generados internamente, siendo aquellos autogenerados o desarrollados por la misma entidad, los mismos que podrán ser considerados como tal cuando tengan un valor de mercado comparable. En este mismo contexto, la norma indica que un activo deber ser identificable, controlado por la empresa y generar beneficios futuros. 
Cristian Homar Blacio-Aguilar; Cecilia Ivonne Narváez-Zurita; Juan Carlos Erazo-Álvarez Ahora bien, la identificabilidad se cumple cuando el activo puede ser transferido por la empresa a través de una venta, arriendo o intercambio, adicionalmente cuando este activo proviene de derechos contractuales o de otros de tipo legal (Ibídem).

Por otro lado, el Plan General de Contabilidad de España, menciona que, para el reconocimiento inicial de un inmovilizado de naturaleza intangible, este debe cumplir con el criterio de identificabilidad, el cual implica ajustarse a uno de los requisitos, es decir, ser separable o surgir de derechos contractuales. En cuanto a los derechos de traspaso, estos podrán figurar en el activo una vez que su valor se ponga en manifiesto mediante una adquisición onerosa, el cual será objeto de amortización y correcciones por deterioro de acuerdo al tratamiento de activos intangibles (Ministerio de Economía y Hacienda, 2007).

Sin embargo (Vargas \& Escobar, 2005), argumentan que algunos activos intangibles pueden encontrarse incluidos, o contener cierta esencia de naturaleza tangible, un claro ejemplo es la documentación legal en caso de una patente, un cd para los artistas o jugadores de fútbol a quienes se les valora por su talento; para ello, es necesario realizar un análisis minucioso para determinar cuál de los dos elementos tiene un peso más relevante y proceder a tratarlo como un activo intangible, un activo biológico o como propiedad, planta - equipo, por cuanto en muchos de los casos el elemento físico es una parte integrante y esencial en el funcionamiento o desarrollo del activo intangible (Sánchez Santos, Castellanos García \& Pena López, 2003).

En cuanto al valor inicial de un activo intangible, son todos los costos directamente relacionados como las remuneraciones a los empleados y honorarios que se deriven directamente de la puesta en marcha y de comprobación del activo, estos costos se comienzan a capitalizar cuando el activo cumple con todos los requisitos de reconcomiendo antes mencionados; en este proceso no se pueden incluir gastos de publicidad o marketing, gastos administrativos, pérdidas por ineficiencias operativas y los gastos de financiamiento ya sea para la adquisición o formación (Fundación IFRS, 2017), en este sentido, (Rubiños, 2016) resalta la importancia de demostrar que un activo cumple las condiciones para ser considerado como intangible, siendo 


\section{Revista Arbitrada Interdisciplinaria KOINONIA \\ Año V. Vol V. $\mathrm{N}^{\circ} 10$. Julio - Diciembre 2020 \\ Hecho el depósito de Ley: FA2016000010 ISSN: 2542-3088 \\ FUNDACIÓN KOINONIA (F.K). Santa Ana de Coro. Venezuela.}

Cristian Homar Blacio-Aguilar; Cecilia Ivonne Narváez-Zurita; Juan Carlos Erazo-Álvarez de otro modo, todos los costos relacionados serán aplicados al resultado del periodo.

Por otro lado, una empresa podrá controlar un activo cuando vaya a obtener beneficios económicos en el futuro, provenientes de los recursos que genere este activo, además de restringir estos beneficios a terceros. La entidad se basará en su propio juicio para determinar las probabilidades de obtener estos beneficios económicos por el uso del activo con la mayor certeza posible, partiendo de la evidencia principal al momento del reconocimiento, dando mayor énfasis a la evidencia de fuentes ajenas o externas (García, 2004).

Como complemento, luego del reconocimiento del activo, está la medición, la cual se realiza en base a dos modelos: el modelo del costo, el modelo de revaluación. Tanto el modelo del costo como el modelo de revaluación indican que el activo se contabilizará por su costo, el valor de revaluación en su debido momento, restando de ellos la amortización acumulada y el importe acumulado de las pérdidas ocasionadas por el deterioro de valor (Ibídem). La NIC 38 señala que el "Importe en libros es el importe por el que un activo se reconoce en el estado de situación financiera, después de deducir la amortización acumulada y las pérdidas por deterioro acumuladas que se refieran al mismo" (IFRS, 2017, p. A1861).

Es también relevante indicar que un activo intangible al tratarse de un activo no financiero posee una vida útil, que es el período durante el cual se espera utilizar el activo o el número de unidades producidas o similares que se pretende obtener del mismo, ya que la entidad será quien determine la vida útil de este activo, que puede ser finita mediante una evaluación de la duración o del número de unidades productivas que constituyan la vida útil del activo, o indefinida cuando luego de realizar un análisis de todos los factores relevantes no se pueda determinar un límite de tiempo en el cual el activo genere entradas de flujo de efectivo neto para la empresa (lbídem).

Por otra parte, para (Ramírez, Baidez \& Lorduy, 2010), existen dos tipos de activos intangibles, los identificables y los no identificables; en el primer grupo, estos autores mencionan que se encuentran aquellos activos intangibles a los que se les puede atribuir facilmente beneficios económicos futuros, por cuanto son separables y la 
Cristian Homar Blacio-Aguilar; Cecilia Ivonne Narváez-Zurita; Juan Carlos Erazo-Álvarez entidad posee derechos legales sobre estos como por ejemplo los gastos de investigación, propiedad industrial, intelectual, entre otros, mientras que los activos intagibles no identificables, son a los que resulta difícil asociarles beneficicios económicos futuros por la naturaleza de su identificación, entre estos se encuentra la clientela, razón social de la empresa, nivel de competencia, prestigio, el capital humano, entre otros.

Mientras tanto la (Superintendencia de Compañias del Ecuador, 2011), mediante la Comisión del proyecto de la aplicación de las NIIF, al realizar una comparación entre las normas completas y la normativa para pymes, indicó que estas últimas en su sección 18, no admiten activos intangibles generados internamente por la empresa, además considera que todos los activos intangibles poseen una vida útil finita, y si una entidad no está en capacidad de determinar la vida útil de este activo, se supondrá que esta será de diez años (Paredes, 2019).

\section{Valoración de jugadores de fútbol en los clubs deportivos}

Los jugadores deben constar en el grupo de los activos intangibles, debido a que se valoran por su talento, mas no de sus servicios o su sueldo, este reconocimiento toma el nombre de derecho federativo, que de acuerdo a (Re \& Seré, 2012) se entiende como el derecho que tiene una institución para inscribir a un determinado deportista en una competición oficial en representación de la misma. Este derecho nace con el fichaje del jugador para un determinado club deportivo mediante un acuerdo contractual en el cual se establecen todos los parámetros, como el precio, tiempo de duración, entre otros (Navascués, 2005).

Por otra parte, se encuentran los jugadores de cantera, los cuales no se consideran como activos intangibles debido a que no cuentan con un valor de adquisición; estos jugadores al ser formados por el club, no poseen un contrato formal que los respalde para ser considerados dentro de este grupo de activos. Es importante también, mencionar a los jugadores que no poseen el derecho de pase de su club anterior hacia el club al cual representan posteriormente. Por otro lado, (García, 2019) menciona una variedad de formas para obtener los derechos de un jugador de un 


\section{Revista Arbitrada Interdisciplinaria KOINONIA \\ Año V. Vol V. N${ }^{\circ} 10$. Julio - Diciembre 2020 \\ Hecho el depósito de Ley: FA2016000010 ISSN: 2542-3088 \\ FUNDACIÓN KOINONIA (F.K). Santa Ana de Coro. Venezuela.}

Cristian Homar Blacio-Aguilar; Cecilia Ivonne Narváez-Zurita; Juan Carlos Erazo-Álvarez

club a otro, entre ellas se encuentran las cesiones temporales, los traspasos, adquisición, mantenimiento y desarrollo de jugadores de cantera, entre otros.

Ahora bien, todas estas formas deben contar con una metodología de valoración para establecer un valor al momento de un traspaso, cesión o cualquier otra modalidad de transferencia de los jugadores de plantilla. Cabe destacar que estos valores en cualquiera de estos casos, significaría un ingreso para la entidad que se desprende de dicho activo, como normalmente sucede en las empresas.

Atendiendo estas consideraciones (Bursesi \& Carratalá, 2003), mencionan el término de derecho econonómico, traducido como el beneficio económico que percibirá una entidad deportiva por la transferencia de un jugador a otro club; en este mismo marco, (Navascués, 2005), lo define como la valuación de los derechos federativos, debido a que estos derechos poseen un contenido patrimonial representado por los derechos económicos derivados de los derechos federativos.

En el caso de la amortización de los derechos federativos de los jugadores, (Bursesi \& Carratalá, 2003), sostienen que esta no debe depender de la edad de los jugadores. Sucede pues, que así no se respeta los plazos legales estipulados en los contratos, que es lo que origina el derecho del club sobre el activo intangible, al vencer el contrato, por defecto se vería amortizado el derecho federativo en su totalidad.

Atendiendo las consideraciones anteriores, adentrándose en metodologías internacionales de valoración de jugadores, (Moreno \& Serrano, 2002), señalan al respecto que en España hubo un debate por el tratamiento contable más adecuado para los gastos incurridos en la formación de los deportistas, el cual consistía, por una parte, en capitalizarlos y considerarlos como activos intangibles de la entidad, y por otra, no capitalizarlos enviándolos directo al resultado del periodo (Mesa Callejas, Osorio Agudelo \& Castaño Ríos, 2016).

En este sentido, las sociedades anónimas deportivas (SAD) de España (Villacorta Hernández, 2006), adoptaron la segunda opción, por cuanto al punto de vista del comité encargado de la adopción, respeta el principio de prudencia, reflejando todas estas erogaciones en el estado de pérdidas y ganancias del periodo. No obstante, todos los desembolsos incurridos para adquisición de derechos, formación y 
Cristian Homar Blacio-Aguilar; Cecilia Ivonne Narváez-Zurita; Juan Carlos Erazo-Álvarez desarrollo de jugadores adquiridos se consideran activos intangibles, cuyo valor se amortizará durante los años de duración del contrato firmado con el jugador (Moreno \& Serrano, 2002).

Debe señalarse que existen también otras metodologías para la valoración de los jugadores de fútbol; como es el método de las jerarquías analíticas (AHP por sus siglas en inglés) una metodología de decisión basada en una variedad de criterios y características cuantitativas como cualitativas en del desempeño de un deportista, tomando en cuenta factores como: asistencias, goles, partidos, edad, sanciones, fallas, entre otras.

De esta manera se asigna un valor al jugador, del cual se espera obtener beneficios económicos para el club y por tal motivo es considerado como un activo intangible o inversión (Valencia, 2017). En este sentido, (Bursesi \& Carratalá, 2003), señalan algunas metodologías y variables para la valoración de un deportista. Costo por criterio de éxito: esta metodología propone asignar todas las erogaciones económicas incurridas en la formación de los jugadores dentro de un ejercicio económico, como el valor origen de los jugadores que llegan a firmar su primer contrato en dicho período.

Por otra parte, cuando no se llegase a habilitar a ningún jugador, estos valores serán considerados como una pérdida del ejercicio. La cuenta de acumulación de estos valores, se denominaría como una cuenta transitoria de costos de formación, la cual al finalizar el período debe ser ajustada a la cuenta correspondiente, ya sea enviando sus valores al resultado del período o de ser el caso al activo, por lo que no debe reflejarse (costos de formación) en los estados financieros.

Costo por órdenes: se realiza una acumulación de los costos incurridos en la formación de cada jugador de manera individual en fichas o kardex, hasta que se realice la suscripción del primer contrato profesional o pase a ser un activo terminado $^{2}$ que pueda ser transferible (derechos de pase). Cuando exista una baja prematura, es decir, que no se dé la habilitación profesional del jugador, todas las

\footnotetext{
${ }^{2}$ Activo terminado: activo generado por la empresa luego de un proceso de transformación, en el caso de un jugador de fútbol, el activo terminado sería el jugador profesional en la plantilla de un club deportivo luego de todo el proceso de formación.
} 
Cristian Homar Blacio-Aguilar; Cecilia Ivonne Narváez-Zurita; Juan Carlos Erazo-Álvarez erogaciones deben reconocerse como una pérdida del ejercicio en que ocurre dicha baja, así como también en el caso de una investigación fracasada.

Costo por grupos / camadas: se reconoce por grupos a los jugadores de cantera 0 de fútbol amateur ${ }^{3}$ como una actividad conjunta, por lo que los costos acumulados se asignan al grupo, el mismo que finalizará el proceso con un número reducido de jugadores, seleccionados por sus méritos y cualidades, con los cuales se va a suscribir su primer contrato. El período de formación de todo el grupo va entre 3 a 5 años, es importante definir este punto para la ejecución del contrato y posterior traspaso de los jugadores.

\section{América latina y el reconocimiento contable de los jugadores del fútbol}

En este sentido (Rezende \& Custodio, 2012), en su análisis mencionan que en los clubes de fútbol en Brasil, los representantes de este sector no han dado la importancia del caso a la adopción de las prácticas contables adecuadas que promulguen la transparencia de la información financiera, por cuanto se revela la prevalecencia de diversidad de criterios contables en eventos similares de estas entidades, sobre lo que establece la norma local, además de imposibilitar la comprabilidad de la información entre clubes.

De acuerdo a (Valencia, 2017) en el caso de Colombia, representan un problema los valores sumamente inflados en las transferencias de jugadores, dado que para ello se priorizan temas mediáticos y no deportivos, en consecuencia estas sobrevaloraciones en el mercado, dificulta a los clubes determinar adecuadamente el valor de transferencia de un jugador que se ajuste y cubra las necesidades de la entidad deportiva.

Por otro lado, en Argentina, la política ha intervenido e influido de manera representatitva en el fútbol, lo que ha convertido a este sector en una mafia (Casa, 2019). Hoy en día, en las transacciones realizadas por estas entidades, intervienen grandes sumas de dinero, especialmente por la compraventa de jugadores, que en base al sistema contable local, son considerados como activos no producidos, cuya

\footnotetext{
${ }^{3}$ Amateur: de acuerdo a la Real Academia Española, es un término utilizado para definir a una persona que realiza cierta actividad o deporte de manera no profesional o por placer. En el caso del fútbol amateur, hace referencia al fútbol no profesional o de novatos.
} 


\section{Revista Arbitrada Interdisciplinaria KOINONIA \\ Año V. Vol V. $\mathrm{N}^{\circ} 10$. Julio - Diciembre 2020 \\ Hecho el depósito de Ley: FA2016000010 ISSN: 2542-3088 \\ FUNDACIÓN KOINONIA (F.K). Santa Ana de Coro. Venezuela.}

Cristian Homar Blacio-Aguilar; Cecilia Ivonne Narváez-Zurita; Juan Carlos Erazo-Álvarez concepción no está conforme con que los jugadores forman parte del activo intangible de los clubes (García, 2019).

De hecho, existe insatisfacción por parte de los usuarios diferentes a los acreedores e inversores, como los asociados, colaboradores, voluntarios, empleados, público en general, por cuanto se podrían interesar en conocer las actividades de la institución, puesto que sus objetivos van más allá, como por ejemplo: conocer la situación financiera de la entidad, a fin de determinar si se están cumpliendo los estatutos y reglamentos en cuanto a la asignación de recursos, valorar la eficiencia, eficacia, la capacidad de responder ante los compromisos asumidos. La constante búsqueda de objetividad en el reconocimiento y medición de los jugadores tanto profesionales como amateurs es un problema latente para quienes elaboran la información contable.

En Ecuador no existe información financiera transparente, los clubes deportivos se encuentran en crisis y han contraido muchas deudas por malos fichajes de jugadores. Cada equipo mantiene su información financiera en reserva por cuanto el Estado no exige su presentación al público; miles de dólares son movidos anualmente en este negocio poco transparente. Por su parte, la Federación Ecuatoriana de Fútbol (FEF) que es el ente regulador de los clubes deportivos no ha planteado hasta el momento ninguna exigencia para regularizar y formalizar la constitución de los mismos como sociedades deportivas que reflejen su situación financiera y sus resultados al final de cada periodo (Casa, 2019).

Por su parte, (Carrión, 2013) indica que en el ámbito administrativo y financiero, no se observan planes estratégicos a mediano plazo, presupuestos aprobados, estados de situación financiera; la contabilidad no es clara, la Federación Ecuatoriana de Fútbol (FEF) no es la excepción, por cuanto no cuenta con la capacidad regulatoria necesaria; (Casa, 2019) menciona que en Ecuador, el registro de la actividad económica y presentación de estados financieros es muy básica; la mayoría de clubes deportivos se encuentran constituidos como entidades sin fines de lucro, por lo que no presentan información a la Superintendecia de Compañías.

En el año 2015, el único club que ha publicado esta información es el Independiente del Valle, que a pesar de aplicar NIIF, dicha información se asemeja más a la 


\author{
Revista Arbitrada Interdisciplinaria KOINONIA \\ Año V. Vol V. $\mathrm{N}^{\circ} 10$. Julio - Diciembre 2020 \\ Hecho el depósito de Ley: FA2016000010 \\ ISSN: 2542-3088 \\ FUNDACIÓN KOINONIA (F.K). Santa Ana de Coro. Venezuela.
}

Cristian Homar Blacio-Aguilar; Cecilia Ivonne Narváez-Zurita; Juan Carlos Erazo-Álvarez contabilidad de una empresa comercial, dejando de lado el hecho de ser una entidad deportiva. En Ecuador existe deficiencia en la contabilización de un jugador, del tratamiento de su vida útil; en la actualidad, para asignar el importe de traspaso de un jugador, se hace de manera empírica, sin ninguna metodología establecida, por cuanto se basa en el poder de negociación de los clubes, en valores de traspasos anteriores y su relevancia en el mercado. El reconocimiento y medición del valor de un jugador de fútbol influye significativamente en la información financiera de una entidad deportiva, la cual, en consecuencia de lo antes mencionado, resulta poco razonable y útil para la toma de decisiones (Ibídem).

\title{
MÉTODO
}

El presente estudio corresponde a una investigación no experimental, que tiene por finalidad proponer procedimientos adecuados para resolver la problemática presente en la información contable y financiera de las entidades deportivas del Ecuador. En cuanto a la perspectiva de la indagación, se realizó mediante un enfoque cualitativo (Aldana Zavala, 2019), tomando en cuenta metodologías para el reconocimiento y medición de los jugadores de fútbol a nivel internacional, partiendo no solo de la normativa contable, sino de perspectivas y criterios basados en la realidad de los clubes deportivos (Erazo y Narváez, 2020).

La intervención consistió en una entrevista realizada a la contadora del Club Deportivo Cuenca. Durante la entrevista la persona encargada respondió las preguntas en base a las respuestas tentativas establecidas para cada interrogante, dando además argumentos personales en algunos de los casos, que apoyaron y fortalecieron la investigación. Las preguntas fueron planteadas con el objetivo de conseguir información acerca de metodologías y procedimientos contables en el reconocimiento y medición del valor de los jugadores del plantel, además de comprobar teorías respecto a la transparencia y razonabilidad de la información financiera.

Adicionalmente, se realizó también, una ficha de revisión documental de algunos clubes del país para conocer detalles de carácter general y particular de cada uno de estos; se procedió revisando información publicada en las respectivas páginas 
Cristian Homar Blacio-Aguilar; Cecilia Ivonne Narváez-Zurita; Juan Carlos Erazo-Álvarez

oficiales de cada equipo y de fuentes externas como la página de la Superintendencia de Compañías, Valores y Seguros, con lo cual se pudo determinar aspectos relevantes.

\section{RESULTADOS}

A continuación, se presentan los resultados obtenidos en base a los instrumentos de investigación aplicados:

El correcto reconocimiento y medición contable de los jugadores de fútbol aporta significativamente a reflejar la realidad y razonabilidad de los estados financieros de una entidad deportiva. La entrevista realizada a la contadora del Club Deportivo Cuenca aportó significativamente a la investigación, ya que corrobora la presencia del problema en los clubes deportivos del Ecuador.

Se ha determinado que el Club Deportivo Cuenca no realiza un correcto reconocimiento y valoración de los jugadores de fútbol del plantel; adicionalmente, se identificó que no se registran como activos intangibles de la entidad. En este mismo contexto, se conoce que los jugadores son considerados y manejados como trabajadores en una empresa común, es decir, todas las erogaciones realizadas en ellos afectan directamente al resultado del período. Por otra parte, al no contar con un valor contable para los traspasos, estos valores se determinan en base a cláusulas contractuales que no poseen metodología alguna, dejando en controversia las variables que hayan sido utilizadas o no para establecer dicho valor.

Por otra parte, mediante la aplicación de la ficha de revisión documental se obtuvo información de ámbito general con respecto a la naturaleza de las instituciones deportivas de Ecuador. Partiendo desde el punto de vista de su constitución y reconocimiento a nivel de contribuyente, se identificó que, en gran parte, los clubes son personas jurídicas.

Personería Jurídica: corresponde al reconocimiento de una empresa, la cual debe asumir una actividad u obligación bajo una responsabilidad jurídica, frente a sí mismo y a los organismos de control; en este sentido, la mayoría de los clubes deportivos ecuatorianos están constituidos como sociedades sin fines de lucro, que 
Cristian Homar Blacio-Aguilar; Cecilia Ivonne Narváez-Zurita; Juan Carlos Erazo-Álvarez bajo la legislación nacional sus acciones no califican como actos de comercio, no obstante, la Ley de Régimen Tributario Interno (LORTI), establece la obligatoriedad de llevar contabilidad. Sin embargo, el hecho de que se obligue a llevar contabilidad, no garantiza un manejo adecuado; la realidad es que la administración de estos clubes se lo hace como una empresa comercial. En consecuencia, no se evidencia la realidad de sus estados financieros, en vista de que no se reporta información a la Superintendencia de Compañías, Valores y Seguros, se desapegan totalmente de la normativa internacional, por cuanto contable y tributariamente este tipo de contribuyentes son muy informales.

Por su parte, el único club que cuenta con información al año 2015 en la plataforma de la Superintendencia de Compañías Valores y Seguros (Independiente del Valle), refleja en sus activos intangibles elementos ajenos al talento de los jugadores de acuerdo a lo detallado en las notas explicativas de la institución, lo que nuevamente pone al descubierto la realidad de la contabilidad deportiva nacional.

\section{PROPUESTA}

Haciendo uso de los resultados obtenidos con los instrumentos de investigación, y con la información obtenida de diferentes fuentes, se ha planteado una guía que servirá a los clubes de fútbol del país, para registrar y valorar adecuadamente a sus jugadores, quienes son los activos intangibles de la empresa. De esta manera, estas instituciones podrán mostrar su información financiera de manera razonable. 
Cristian Homar Blacio-Aguilar; Cecilia Ivonne Narváez-Zurita; Juan Carlos Erazo-Álvarez

\begin{tabular}{|c|c|c|c|}
\hline Grupo & Subgrupo & Código & Cuenta \\
\hline \multirow{5}{*}{ Activo } & \multirow{5}{*}{$\begin{array}{l}\text { Activos } \\
\text { intangibles }\end{array}$} & 1020407 & Jugadores en formación \\
\hline & & 1020408 & $\begin{array}{l}\text { jugadores y/o activos intangibles } \\
\text { formados internamente }\end{array}$ \\
\hline & & 1020409 & $\begin{array}{l}\text { Amortización acumulada de jugadores } \\
\text { adquiridos }\end{array}$ \\
\hline & & 1020410 & $\begin{array}{l}\text { Jugadores y/o Activos intangibles } \\
\text { adquiridos }\end{array}$ \\
\hline & & 1020411 & $\begin{array}{l}\text { Amortización acumulada de jugadores } \\
\text { formados internamente }\end{array}$ \\
\hline Patrimonio & $\begin{array}{c}\text { Otros } \\
\text { resultados } \\
\text { integrales }\end{array}$ & 30503 & $\begin{array}{l}\text { Ganancia en revalorización de activos } \\
\text { intangibles }\end{array}$ \\
\hline \multirow{3}{*}{ Gastos } & Otros gastos & 610403 & $\begin{array}{l}\text { Pérdida en revalorización de activos } \\
\text { intangibles }\end{array}$ \\
\hline & $\begin{array}{l}\text { Gastos en } \\
\text { procesos de } \\
\text { formación }\end{array}$ & 61012404 & Gastos en formación de jugadores \\
\hline & Amortizaciones & 61012201 & Amortización en valor jugadores \\
\hline
\end{tabular}

Figura 1. Catálogo de cuentas para reconocimiento y medición de intangibles en clubes de fútbol. Fuente: Investigadores, 2020.

Adicionalmente, como parte introductoria a la presente guía, se ha detallado un mini catálogo exponiendo las cuentas recomendadas para una mejor identificación y aplicación de las mismas en los estados financieros de las entidades.

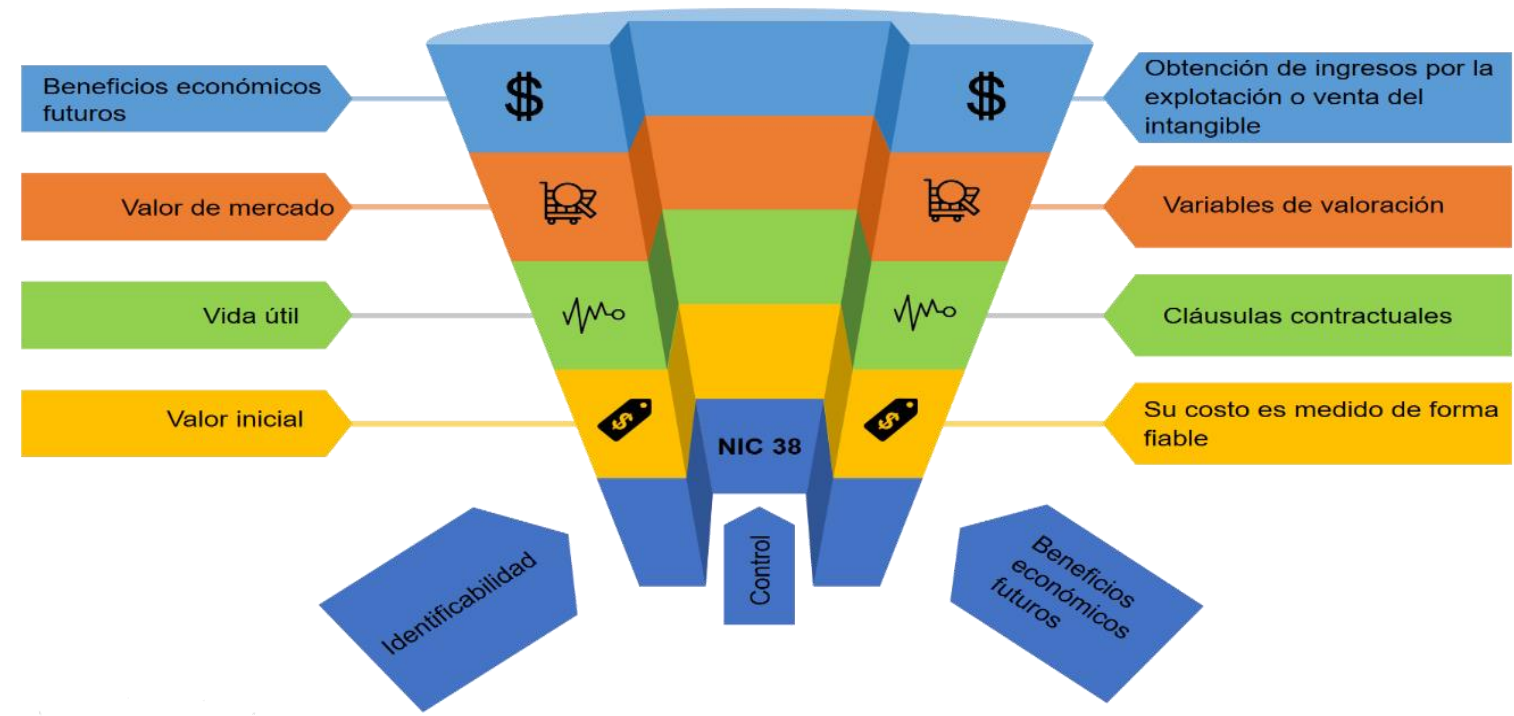

Figura 2. Componentes de los activos intangibles en los clubes de fútbol (jugadores). Fuente: Investigadores, 2020. 
Cristian Homar Blacio-Aguilar; Cecilia Ivonne Narváez-Zurita; Juan Carlos Erazo-Álvarez

En la figura 2 se aprecian los componentes y las características de los activos intangibles que se han venido estudiando, todos estos son elementos derivados de la investigación y constituyen la base para el desarrollo de esta propuesta. Cada uno de los componentes deberá determinarse de acuerdo a lo que indica la figura 3 , en la cual se detallan las diferentes fases comprendidas en las etapas de reconocimiento y medición de este grupo de activos.

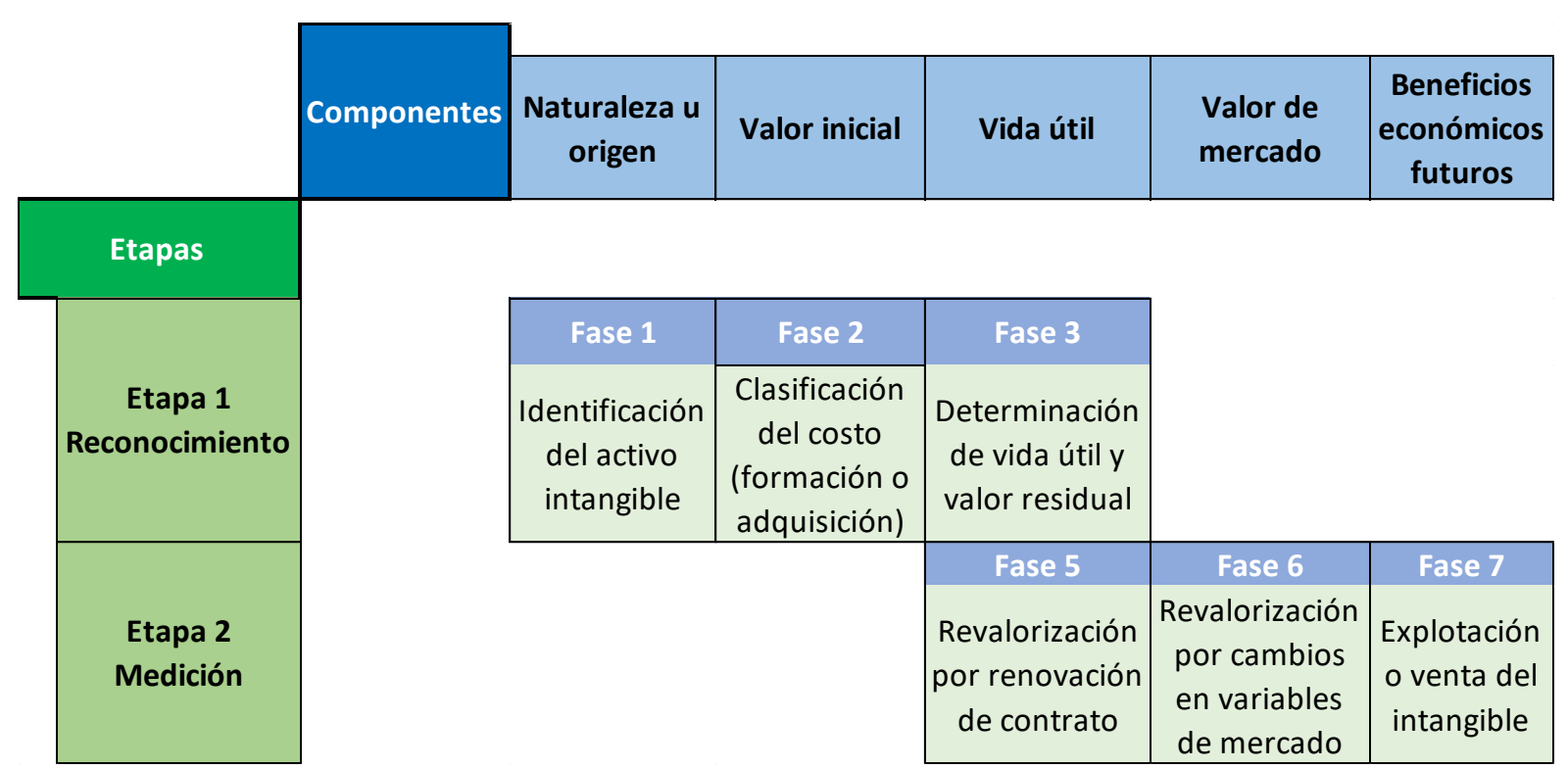

Figura 3. Fases del reconocimiento y medición de los activos intangibles en los clubes de fútbol según la NIC 38 y metodologías internacionales. Fuente: Investigadores, 2020.

\section{Etapa 1: Reconocimiento}

Identificación del tipo de activo intangible: basándose en lo que indican la NIC 38 y los autores citados en el presente trabajo, existen dos tipos de activos intangibles de acuerdo a su origen o naturaleza:

- Los primeros son los intangibles adquiridos; para la presente propuesta, se identificarán en este grupo a los jugadores que fueron obtenidos o traspasados desde otro club deportivo, para lo cual existió un contrato legal de por medio. Este contrato contendrá varias cláusulas, entre las cuales: su 
Cristian Homar Blacio-Aguilar; Cecilia Ivonne Narváez-Zurita; Juan Carlos Erazo-Álvarez valor de mercado, el tiempo de duración de contrato, además se recomienda adjuntar un histórico del reconocimiento y medición de estos activos, con el objetivo de transparentar la información financiera y contable para un mejor manejo por parte de la empresa adquiriente. La cuenta contable a utilizar para este tipo de intangibles sería activos intangibles adquiridos o jugadores adquiridos.

\begin{tabular}{|c|c|c|c|}
\hline \multicolumn{2}{|c|}{$\begin{array}{l}\text { Código Cuenta } \\
1020410 \text { Jugadores adquiridos }\end{array}$} & Debe & Habe \\
\hline \multicolumn{2}{|c|}{1020410 Jugadores adquiridos } & $X X X$ & \\
\hline 110101 & Bancos & & $x x x$ \\
\hline$P / r$ & uisición de jugado & & \\
\hline
\end{tabular}

Figura 4. Asiento para adquisición de jugadores. Fuente: Investigadores, 2020.

- Los activos intangibles generados internamente, hace referencia a los jugadores de la cantera del club deportivo, es decir, aquellos que han sido formados por la institución desde una corta edad y que han llegado a formar parte de la plantilla principal del equipo. En este grupo, es muy importante tener en cuenta que, para que estos jugadores lleguen a ser jugadores profesionales, la institución invirtió ciertos valores en su formación (ver figura $5)$.

Clasificación de los costos: es importante que las entidades reconozcan y clasifiquen adecuadamente los desembolsos realizados en sus jugadores, para ello, se debe tener presente las variables mencionadas en el punto anterior.

- En primer lugar, para los jugadores de cantera, se deberá acumular todos los valores implicados en la formación de estos en una cuenta transitoria denominada jugadores en formación (ver figura 5), con el fin de que al final del periodo esta cuenta se pueda reclasificar, ya sea dentro del grupo de activos intangibles a la cuenta contable jugadores y/o activos intangibles formados internamente (ver figura 6), o aplicarse al resultado del periodo en una cuenta de gastos en formación de jugadores (ver figura 7).

- Para este proceso, se recomienda una combinación entre los siguientes métodos, costo por criterio de éxito y costo por grupos / camadas por ser los 
Cristian Homar Blacio-Aguilar; Cecilia Ivonne Narváez-Zurita; Juan Carlos Erazo-Álvarez más adecuados para el medio deportivo nacional. Dentro de este marco, la metodología propuesta recomienda asignar los costos de formación de todo el conjunto de jugadores amateur a los jugadores que efectivamente logren firmar un contrato de fútbol profesional con el club deportivo, teniendo en cuenta que el periodo de formación de los jugadores oscila entre los 3 y 5 años, para lo cual será necesario crear una cuenta transitoria dentro del activo para acumular todos estos valores. Cuando un jugador forme parte de la plantilla profesional, este valor correspondería al valor inicial del activo intangible.

\begin{tabular}{|c|c|c|c|}
\hline Código & Cuenta & Debe & Haber \\
\hline \multicolumn{2}{|c|}{1020407 Jugadores en formación } & Xxx & \\
\hline 110101 & Bancos & & $X X X$ \\
\hline$P / r$ & mación de cantera & & \\
\hline
\end{tabular}

Figura 5. Asiento para acumulación de erogaciones en formación de jugadores amateurs. Fuente: Investigadores, 2020.

\begin{tabular}{|c|c|c|c|}
\hline Código & Cuenta & Debe & Haber \\
\hline \multicolumn{2}{|c|}{1020408 Jugadores formados internamente } & $x x x$ & \\
\hline 1020407 & Jugadores en formación & & $x x x$ \\
\hline$P / \mathbf{r}$ & nación de valores transitorios a & tangib & \\
\hline
\end{tabular}

Figura 6. Asiento de reclasificación de activos. Fuente: Investigadores, 2020.

En el caso de que ninguno de los jugadores del grupo llegue a formar parte de la plantilla principal, todos estos valores acumulados, se aplicarán al resultado del periodo en la cuenta antes mencionada.

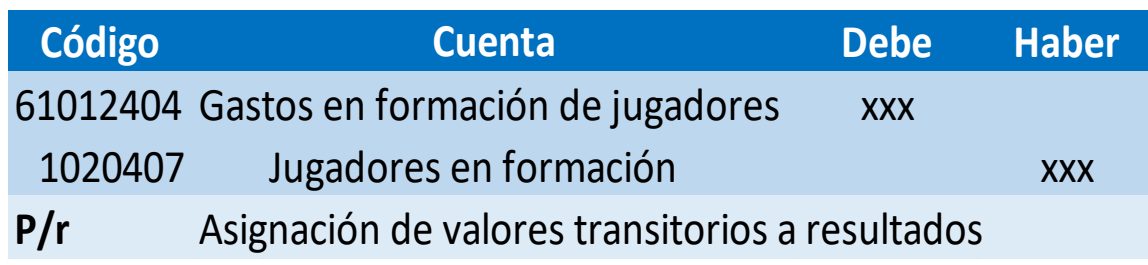

Figura 7. Asiendo de afectación a resultados del periodo. Fuente: Investigadores, 2020. 
Cristian Homar Blacio-Aguilar; Cecilia Ivonne Narváez-Zurita; Juan Carlos Erazo-Álvarez

- Para los jugadores adquiridos, la metodología propuesta establece que se asignen al activo intangible los valores de adquisición y cualquier otro valor en que se incurra para poner en total forma y disponibilidad a los jugadores para ser parte de la plantilla que va a representar al equipo en campeonatos tanto nacionales como internacionales. Para este caso los valores incurridos en la adquisición y preparación del jugador se deberán aplicar directamente a la cuenta del intangible.

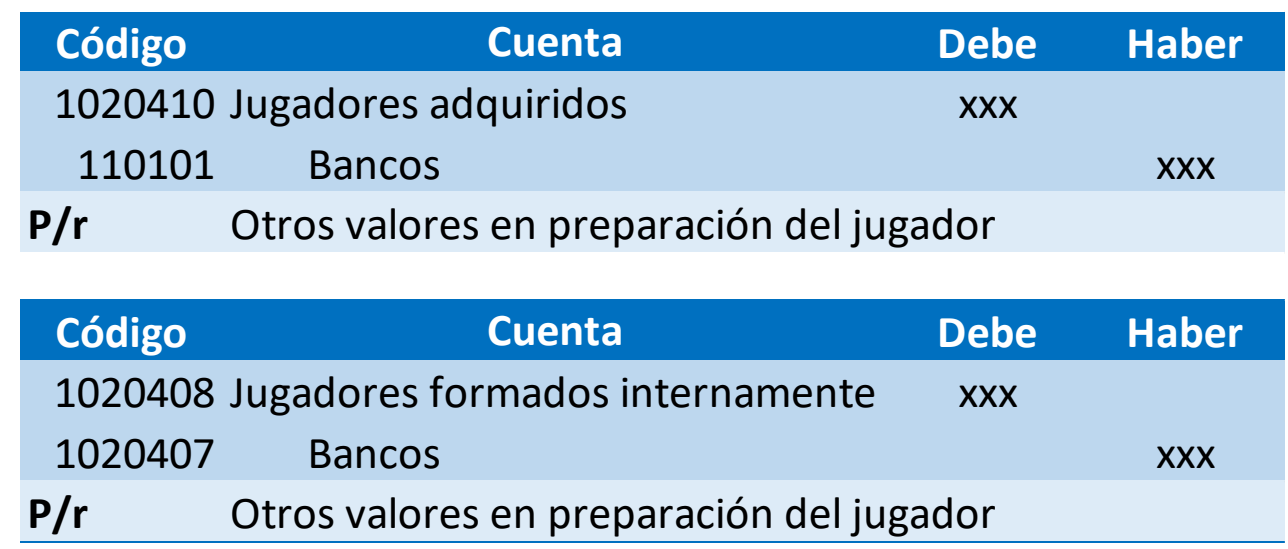

Figura 8. Aplicación de valores incurridos para puesta en marcha de los activos. Fuente: Investigadores, 2020.

Es importante aclarar que la figura 8 muestra la contabilización de valores incurridos en los jugadores antes de que se encuentren habilitados en la plantilla principal del club, es decir, que siguen siendo una inversión en los jugadores, las demás erogaciones para acondicionamiento, entrenamiento, capacitaciones, evaluaciones, entre otras, que formen parte de las operaciones y procesos con la plantilla deberán aplicarse al resultado del periodo.

La vida útil del activo intangible se basará en las cláusulas estipuladas en el contrato, sea este de adquisición o pase de cantera a plantilla principal. Su valor residual se determinará de acuerdo al valor de mercado de otros jugadores, de acuerdo al tiempo del contrato y al desempeño en cada año, por lo que puede ser reajustable al final del periodo fiscal.

Dentro del mismo contexto, el contrato deberá establecer una cláusula que permita o no la renovación del mismo; con esto, se alargaría la vida útil del intangible, derivando además una revalorización del activo; este proceso partirá del valor 
Cristian Homar Blacio-Aguilar; Cecilia Ivonne Narváez-Zurita; Juan Carlos Erazo-Álvarez

residual en la fecha de renovación. Para las amortizaciones, se utilizará la cuenta contable amortización de jugadores adquiridos 0 amortización de jugadores formados internamente; es importante mantener la clasificación para una correcta identificación de los valores que se van a aplicar al resultado del periodo.

\begin{tabular}{|c|c|c|c|}
\hline Código & Cuenta & Debe & Haber \\
\hline 61012201 & Amortización en valor jugadores & $x X X$ & \\
\hline 1020409 & $\begin{array}{l}\text { Amortización acumulada de } \\
\text { jugadores adquiridos }\end{array}$ & & $X X X$ \\
\hline$P / r$ & Amortización de intagibles & & \\
\hline
\end{tabular}

Figura 9. Asiento de contabilización de amortizaciones al final del periodo. Fuente: Investigadores, 2020.

\section{Etapa 2: Medición}

En la segunda etapa de la propuesta, se encuentra la asignación de un valor a los activos intangibles, para esta fase, en el caso de los jugadores amateurs se deberá realizar un análisis de los valores actuales de mercado de los jugadores que recientemente han entrado al fútbol profesional para realizar una comparativa, de ser necesario reajustar su valor inicial, siempre y cuando este sea superior al calculado por la institución que se trate de un valor razonable, ya sea por la demanda del momento, o por el potencial del deportista (ver figura 11).

Para los jugadores adquiridos, el valor convenido en el contrato deberá ajustarse al valor razonable del mercado para evitar revalorizaciones a corto plazo. Dentro de este proceso deberá tomar en cuenta las respectivas amortizaciones acumuladas a la fecha del revalúo; estos ajustes deben ser consideradas de acuerdo a cada caso, en primer lugar, si la revalorización genera un superávit en el valor del jugador será clasificado y registrado como otros resultados integrales en la cuenta ganancia en revalorización de activos intangibles; por otra parte, si esta revaluación ocasiona una pérdida de valor del activo, será registrada en el resultado del periodo como pérdida en revalorización de activos intangibles.

Poniendo en contexto el punto anterior y suponiendo que tenemos los siguientes datos de un jugador: 
Cristian Homar Blacio-Aguilar; Cecilia Ivonne Narváez-Zurita; Juan Carlos Erazo-Álvarez

$\begin{array}{lr}\text { Valor de adquisición del jugador } & 1,000.00 \\ \text { Amortización acumulada } & -200.00 \\ \text { Valor en libros } & 800.00 \\ \text { Valor de mercado } & 1,200.00 \\ \text { Ganancia } & 400.00 \\ \text { Aumento en valor del jugador } & 200.00\end{array}$

La ganancia se obtiene de la diferencia entre el valor en libros y el valor de mercado, mientras que el aumento en el valor del jugador, nace de la diferencia entre su valor de adquisición y el valor de mercado. En base a este análisis, el asiento sería el siguiente:

\begin{tabular}{llll}
\multicolumn{1}{l}{ Código } & \multicolumn{1}{c}{ Cuenta } & Debe & Haber \\
1020410 & Jugadores adquiridos & 200.00 & \\
1020409 & $\begin{array}{l}\text { Amortización acumulada de } \\
\text { jugadores adquiridos }\end{array}$ & 200.00 & \\
30503 & $\begin{array}{l}\text { Ganancia por revalorización } \\
\text { de intangibles }\end{array}$ & 400.00 \\
P/r & Ganancia en revalorización de intangibles &
\end{tabular}

Figura 10. Asiento de ejemplificación de ganancia por revalorización. Fuente: Investigadores, 2020.

Por otro lado, si el escenario es opuesto, se genera una pérdida en la revalorización del intangible, y se ejemplifica de la siguiente manera.

$\begin{array}{lr}\text { Valor de adquisición del jugador } & 1,000.00 \\ \text { Amortización acumulada } & -200.00 \\ \text { Valor en libros } & =800.00 \\ \text { Valor de mercado } & 600.00 \\ \text { Pérdida } & -200.00 \\ \text { Disminución en valor del jugador } & -400.00\end{array}$


Cristian Homar Blacio-Aguilar; Cecilia Ivonne Narváez-Zurita; Juan Carlos Erazo-Álvarez El procedimiento para determinar estos valores es el mismo que se utiliza para determinar la ganancia y aumento en el valor, por lo tanto, el asiento quedaría de la siguiente manera:

\begin{tabular}{|c|c|c|c|}
\hline & Cuenta & Debe & Haber \\
\hline 610403 & $\begin{array}{l}\text { Pérdida en revalorización de activos } \\
\text { intangibles }\end{array}$ & 200.00 & \\
\hline 1020409 & $\begin{array}{l}\text { Amortización acumulada de jugadores } \\
\text { adquiridos }\end{array}$ & 200.00 & \\
\hline 1020410 & Jugadores adquiridos & & 400.00 \\
\hline
\end{tabular}

Figura 11. Asiento de ejemplificación de la pérdida por revalorización. Fuente: Investigadores, 2020.

Para finalizar, la revalorización de los jugadores será esencial tanto al momento de una renovación de contrato o de acuerdo a su desempeño, lo cual mejorará o no sus cláusulas contractuales y lo más importante para la contabilidad, su valor de mercado. Esta revalorización deberá apegarse a las variables de mercado y variables que menciona la metodología de jerarquías analíticas (AHP), la cual toma en cuenta tanto factores cualitativos como cuantitativos en el desempeño del deportista para establecer un nuevo valor a su talento.

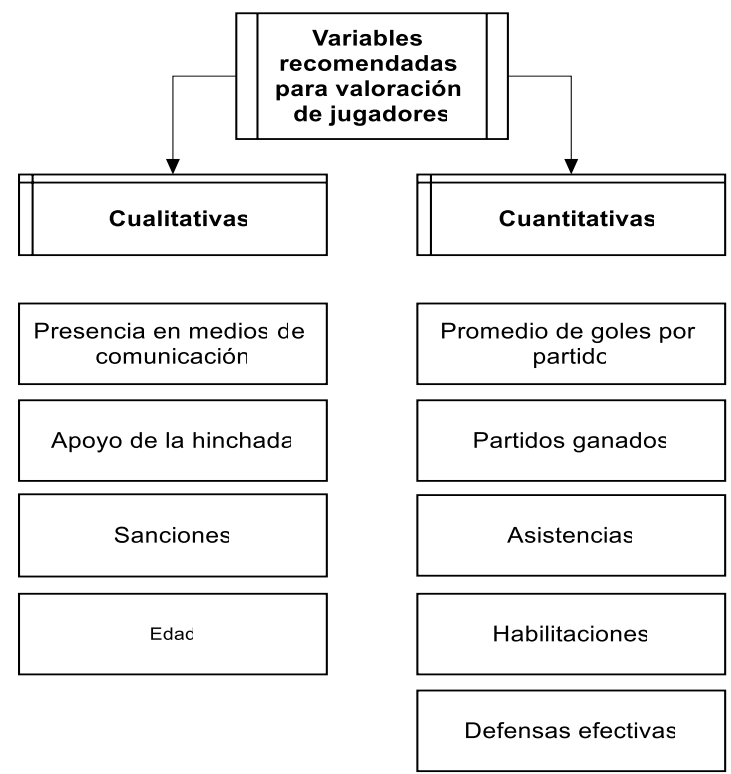

Figura 12. Variables de revalorización. Fuente: Investigadores, 2020. 
Cristian Homar Blacio-Aguilar; Cecilia Ivonne Narváez-Zurita; Juan Carlos Erazo-Álvarez Adicionalmente, se deberán tomar en cuenta las variables que a criterio del profesional sean relevantes en el proceso de valorización del deportista. La aplicación de todo este procedimiento, conllevará a que la información contable y financiera de los clubes deportivos del Ecuador sea confiable, además de transparentar las transacciones entre estas entidades, evitando contratos sobrevalorados e información poco razonable.

\section{DISCUSIÓN}

Retomando el aporte de (Mesén, 2007) a nivel mundial, se considera que la principal razón de aplicación de las NIIF es fomentar la transparencia de la información y un lenguaje común para suprimir las diferencias en su interpretación contable. Esta normativa en el Ecuador mantiene una aplicación parcial e incompleta en la mayoría de empresas, y totalmente nula en muchas otras; esto se debe a que no existen a nivel nacional metodologías, manuales o guías que aporten a una mejor interpretación de las NIIF y de esta manera ajustar los procesos y métodos adecuados a las circunstancias sociales, legales y económicas del país.

La NIC 38 indica cuál es el procedimiento para el reconocimiento y medición de los activos intangibles, sin embargo, no detalla cómo manejar de forma particular a activos como los jugadores de un club de fútbol, para los cuales debe existir una metodología ajustada a su naturaleza y operatividad. En la actualidad, todos los clubes de fútbol nacional llevan una contabilidad comercial de sus transacciones, que, en cierta parte estaría correcto para sus transacciones de ámbito general, mas no para el tratamiento de sus intangibles.

Desde la perspectiva societaria, los clubes deportivos deberían constituirse como sociedades anónimas deportivas, una modalidad que ya se está aplicando en algunos países. Hoy en día casi la totalidad de los clubes han sido constituidos como sociedades sin fines de lucro, por lo cual no figuran de manera adecuada en la economía nacional; las entidades deportivas pertenecen a una industria muy rentable y de alta generación de ingresos en muchos de los casos, es por ello la necesidad de palpar esa información. La Superintendencia de Compañías Valores y Seguros sería la encargada de habilitar esta nueva modalidad a la que estas 
Cristian Homar Blacio-Aguilar; Cecilia Ivonne Narváez-Zurita; Juan Carlos Erazo-Álvarez instituciones puedan acogerse, mantener una estructura organizacional más idónea, además que toda su información contable y financiera se volvería pública.

Los resultados obtenidos demuestran la falta de transparencia en el manejo de la contabilidad, en la compra-venta de jugadores y por lo tanto, en la valoración de los mismos en los clubes deportivos del Ecuador. Por el motivo de no contar con una guía a la que estas instituciones puedan regirse, su información no es razonable y no cumple con el principio de comparabilidad que la normativa internacional pretende.

Las metodologías planteadas en la propuesta de este trabajo, son resultado de un estudio y análisis de técnicas aplicadas en países europeos, donde existe un nivel deportivo y metodológico más avanzado para valorizar sus activos intangibles, por lo que resulta adecuado replicar los procesos más idóneos para los clubes locales.

La presente propuesta, contribuye en la correcta aplicación de la NIC 38 en complemento con métodos internacionales, planteando lineamientos para identificar a los intangibles, clasificarlos y emplear un correcto procedimiento al momento del registro de todos los valores derivados de la formación, adquisición, preparación y revalorización de los jugadores de los clubes deportivos de fútbol; con el fin de presentar en forma razonable la información financiera.

Para mejorar el entendimiento de la propuesta, se plantearon cuentas, asientos, ejemplificaciones que detallan el procedimiento contable, además de las consideraciones a nivel de grupos de activo, patrimonio y gastos en los que tiene incidencia el reconocimiento y medición de los jugadores de fútbol. Entre estos asientos resaltan los referentes a la revalorización de jugadores, por la situación actual de las instituciones en Ecuador el primer paso sería la revalorización de su plantilla.

Esta guía, servirá de base para que, en un futuro cercano, la contabilidad deportiva nacional tome un nuevo rumbo y un mayor protagonismo en la valorización de los jugadores.

\section{FINANCIAMIENTO}

No monetario 
Cristian Homar Blacio-Aguilar; Cecilia Ivonne Narváez-Zurita; Juan Carlos Erazo-Álvarez

\section{AGRADECIMIENTOS}

A las autoridades del Club Deportivo Cuenca, por el apoyo en el desarrollo de la investigación.

\section{REFERENCIAS CONSULTADAS}

Aldana Zavala, J. J. (2019). La competencia epistemológica en el investigador social universitario venezolano. [Epistemological competence in the Venezuelan university social researcher]. Praxis, 15(1), 103-115. https://doi.org/10.21676/23897856.3091

Barrios del Pino, I., Correa, A., Acosta, M., \& González, A. (2003). El concepto de activo intangible y sus tipologías: una revisión de la normativa contable nacional e internacional. [The intangible asset concept and its typologies: a review of national and international accounting regulations]. Recuperado de https://n9.cl/di8v

Benítez Rochel, J., \& Lacomba Arias, B. (2012). Los avances en la valoración económica del deporte en Europa. [Advances in the economic valuation of sport in Europe]. Estudios de economía aplicada, 30(2), 637-654. Recuperado de https://n9.cl//f6e

Bursesi, N., \& Carratalá, J. (2003). Valoracion de los activos intangibles en entidades deportivas: Clubes de fútbol. [Valuation of intangible assets in sport entities: Soccer clubs]. Recuperado de https://n9.cl/flyz

Carrión Mena, F. (2013). La Crisis Del Fútbol Ecuatoriano: El Populismo Financiero Futbolístico. [The Ecuadorian Soccer Crisis: Soccer Financial Populism]. Polémika, 4(10). Recuperado de https://n9.cl/xo38

Casa, J. (2019). La estructura financiera de los clubes de fútbol basados en la perspectiva de valoración del jugador. [The financial structure of soccer clubs based on the player's valuation perspective]. Recuperado de https://n9.cl/0vz7

Collado Comes, J. J. (2012). Impacto socio-económico de los grandes eventos. El caso de Brasil. [Socio-economic impact of major events. The case of Brazil]. Recuperado de https://n9.cl/halu0

Coremberg, A; Sanguinetti, J; Wierny, M. (2016). El fútbol en la economía argentina. Números sin pasiones. [Soccer in the Argentine economy. Numbers without passions]. Universitat de Valencia; Journal of Sports Economics \& Managament; 6; 1; 6-2016; 46-68. Recuperado de https://n9.cl/f1/4 
Cristian Homar Blacio-Aguilar; Cecilia Ivonne Narváez-Zurita; Juan Carlos Erazo-Álvarez

Erazo Álvarez, J. C., \& Narváez Zurita, C. I. (2020). Medición y gestión del capital intelectual en la industria del cuero - calzado en Ecuador. [Measurement and management of intellectual capital in the leather industry - footwear in Ecuador]. Revista Arbitrada Interdisciplinaria Koinonía, 437-467. http://dx.doi.org/10.35381/r.k.v5i9.662

Espinoza Carrión, D. I., Narváez Zurita, C. I., Erazo Álvarez, J. C., \& Machuca Contreras, M. d. (2019). La estrategia financiera como herramienta funcional para la gestión de liquidez en las organizaciones. [Financial strategy as a functional tool for liquidity management in organizations]. Cienciamatria, 502532. https://doi.org/10.35381/cm.v5i1.278

Fundación IFRS. (2018). El Marco Conceptual para la Información Financiera. [The Conceptual Framework for Financial Information]. Recuperado de https://n9.cl/f6ztg

García Pacheco, I. (2015). La fiscalidad de los deportistas de fútbol profesional en el ámbito internacional. [Taxation of professional soccer athletes in the international field]. Recuperado de https://n9.cl/0l5d

García-Parra, M. (2004). Activos inmateriales y contabilidad. [Intangible assets and accounting]. Intangible Capital, 0(3), 214-225. http://dx.doi.org/10.3926/ic.25

García, C. A. (2019). Sociedades Anónimas Deportivas como modelo de gestión de clubes: viabilidad en Argentina y las experiencias en el Derecho Comparado. [Sports Public Limited Companies as a club management model: viability in Argentina and experiences in Comparative Law]. Revista Justicia y Derecho. 2(1), 14-29. Recuperado de https://n9.cl/fi4g

García-del-Barrio, P., \& Pujol, F. (2007). El papel del fútbol en la sociedad actual. Fútbol: ocio y negocio. [The role of soccer in today's society. Soccer: leisure and business.]. Empresa y Humanismo, 102-103. Recuperado de https://n9.cl/3kc2

González, M., Cuenca , M., Higuerey, Á., \& Villanueva , J. (2017). Adopción NIIF en Ecuador: Análisis pre y post sobre magnitudes contables de empresas cotizadas. [IFRS adoption in Ecuador: Pre and post analysis on accounting figures of listed companies.]. X-pedientes Económicos, v. 1, n. 1, p. 30-41. Recuperado de https://n9.cl/in9w

Marcotrigiano Z., L. A. (2011). Discusión del concepto de "activo" dentro del Marco Conceptual de las Normas Internacionales de Información Financiera. [Discussion about the concept of "asset" within the Conceptual Framework of International Financial Reporting Standards ]. Recuperado de https://n9.cl/0nla 
Cristian Homar Blacio-Aguilar; Cecilia Ivonne Narváez-Zurita; Juan Carlos Erazo-Álvarez

Mesa Callejas, R. J., Osorio Agudelo, J. A. \& Castaño Ríos, C. E. (2016). Economía, gestión y fútbol: de la pasión a la sostenibilidad financiera. [Economy, management and football: from passion to financial sustainability]. Borradores Departamento de Economía, (57), 1-21. Recuperado de https://n9.cl/cxxcz

Mesén F., V. (2007). Aplicaciones prácticas de las Normas Internacionales de Información Financiera. [Practical applications of the International Financial Reporting Standards]. [1era ed.]. Costa Rica: Ediciones Tecnológicas.

Ministerio de Economía y Hacienda. (2007). Plan General de Contabilidad. [General accounting plan]. España.

Moreno Rojas, J. y Serrano Domínguez, F. (2002). La activación de los derechos de formación de jugadores en las Sociedades Anónimas Deportivas: una propuesta a la luz de la nueva normativa FIFA. [The activation of the players' training rights in Sports Public Limited Companies: a proposal in light of the new FIFA regulations]. AECA: Revista de la Asociación española de Contabilidad y A. Recuperado de https://n9.cl/uk8qt

Navascués, H. (2005). Fútbol profesional. [Professional soccer].Montevideo, Uruguay: Ediciones de la Plaza

Paredes, J. (2019). Guía NIIF para directores 2019/2020. [IFRS guide for directors 2019/2020]. Recuperado de https://n9.cl/7ka0

Pérez Rico, C., Méndez Rojas, V., Fernández García, C., Alvarado Riquelme, M., \& Méndez Rojas, P. (2017). Comunidad Andina de Naciones (CAN), Perú, Colombia, Bolivia y Ecuador: proceso de convergencia y adopción de las IFRS. [Andean Community of Nations (ACN), Peru, Colombia, Bolivia and Ecuador: IFRS process of convergence and adoption]. Revista Economía y Política, 21(21), 65 - 74. https://doi.org/10.25097/rep.n21.2015.05

Quizhpi Barbecho, R. d., Narváez Zurita, C. I., \& Erazo Álvarez, J. C. (2019). La gestión contable de las empresas comerciales en el marco de las NIIF para PYMES. [Accounting management of commercial companies under the IFRS for PYMES]. Revista Arbitrada Interdisciplinaria Koinonía, 265-298. http://dx.doi.org/10.35381/r.k.v4i2.475

Ramírez Córcoles, Y., Baidez González , A., \& Lorduy Osés , C. (2010). Tratamiento contable de los activos intangibles: una visión internacional. [Accounting treatment of intangible assets: an international vision]. Recuperado de https://n9.cl/l2ib

Re Cardillo, M., \& Seré Santiago, J. R. (2012). La importancia de la aplicación de las normas contables adecuadas enmarcadas en el proceso de profesionalización que atraviesan tanto el Club Atlético Peñarol como el Club Nacional de Football. [The importance of applying the appropriate accounting standards 
Cristian Homar Blacio-Aguilar; Cecilia Ivonne Narváez-Zurita; Juan Carlos Erazo-Álvarez

framed in the professionalization process that both Atlético Peñarol Club and the National Football Club experience]. Montevideo, Uruguay.

Rezende, AJ, \& Custodio, R. dos S. (2012). Análisis de la evidencia de derechos federativos en los estados financieros de los clubes de fútbol brasileños. [Analysis of the evidence of federative rights in the financial statements of Brazilian soccer clubs]. Revista de educación e investigación contable (REPeC), 6 (3). https://doi.org/10.17524/repec.v6i3.235

Rubiños (2016). Norma Internacional de Contabilidad N³8: Activos Intangibles. [International Accounting Standard No. 38 - Intangible Assets]. Recuperado de https://n9.cl/eamhi

Sánchez Santos, J. M., Castellanos García, P., \& Pena López , J. A. (2003). Economía, fútbol y bienestar social. [Economy, soccer and social welfare]. Recuperado de https://n9.cl/4cef

Superintendencia de Compañias del Ecuador. (2011). Adopción de las NIIF en Ecuador. [Adoption of IFRS in Ecuador]. Recuperado de https://n9.cl/8hni

Ugalde Binda, N. (2014). Las Normas Internacionales de Información Financiera: historia, impacto y nuevos retos de la IASB. [International Financial Reporting Standards: history, impact and new challenges of the IASB]. Revista De Ciencias Económicas, $32(1)$ 205-216. https://doi.org/10.15517/rce.v32i1.15058

Valencia Rojas, D. (2017). Aplicación del modelo AHP en la valoración de futbolistas profesionales: Caso James Rodríguez. [Application of the AHP model in the valuation of professional soccer players: James Rodríguez case].Recuperado de https://n9.cl/crim

Vargas Melgarejo, A., \& Escobar Ovalle, L. J. (2005). Análisis de la adopción y/o armonización de la NIC 38 teniendo como base el tratamiento de activos intangibles en México. [Analysis of the adoption and / or harmonization of IFRS 38 based on the treatment of intangible assets in Mexico]. Recuperado de https://n9.cl/m6li

Villacorta Hernández, M. Á. (2006). Reconocimiento contable de los pagos a deportistas profesionales. [Accounting recognition of payments to professional athletes]. Recuperado de https://n9.cl/zmwa 